\title{
PET ENGENHARIA ELÉTRICA CEFET-MG CAMPUS NEPOMUCENO - CONSTRUINDO UM MODELO DE SUCESSO
}

Leonardo S. Ricardino - leonardoricardino119@ gmail.com

Centro Federal de Educação Tecnológica de Minas Gerais, Departamento de Elétrica

Rua Vice Prefeito Walter Mansur, 604 - Bairro Santo Antônio

37250-000 - Nepomuceno - MG

Iago M. Viela - iagomvilela@yahoo.com

Rua José Normando Costa, 210 - Bairro Aeroporto

37205-291 - Lavras - MG

Breno M. Lima - massonbreno1991@gmail.com

Rua João Inácio Dias, 1122 - Centro

37250-000 - Nepomuceno - MG

Ualifer A. da Silva - ualifer@gmail.com

Rua Pedro Correia de Souza, 57 - Bairro Vila Menezes

37250-000 - Nepomuceno - MG

Reginaldo B. Fernandes - reginaldo@ cefetmg.br Rua Nicolau Bauth, 142 - Bairro Santa Filomena 37203-712 - Lavras - MG

Resumo: Objetivando atender a demandas oriundas da universidade e da sociedade, o programa de educação tutorial, do curso de engenharia elétrica do CEFET-MG Campus Nepomuceno está em seu terceiro ano de atuação. A experiência acumulada ao longo desse tempo se mostrou fundamental para que em 2019 o grupo viesse a apresentar um plano de atuação mais ambicioso e inovador. Dentre as atividades desenvolvidas estão palestras e minicursos ministrados, eventos organizados e projetos de pesquisa. Algumas dessas atividades já existiam anteriormente e foram repensadas para que pudesse atingir um maior público e uma melhor eficiência. Os resultados aqui descritos revelam que a atuação do grupo tem sido fundamental para o desenvolvimento da comunidade interna e externa do CEFETMG Nepomuceno.

Palavras-chave: Grupo PET. Engenharia Elétrica. Ensino. Pesquisa. Extensão.

\section{INTRODUÇÃO}

Conforme destaca Rosa Maria et al (2006), a universidade é uma instituição responsável pela formação profissional e científica que tem como objetivo a conservação e progresso dos diversos ramos do conhecimento. Sua organização e seus métodos são pensados, e constantemente modificados, de forma a servir aos interesses da sociedade. Nesse contexto, 
surge em 1979 o Programa de Educação Tutorial (PET), um modelo aplicado ao ensino superior das universidades Brasileiras que visa estimular ensino, pesquisa e extensão através de projetos executados paralelamente aos respectivos cursos.

Quando o PET é implementado dentro da instituição, um ou mais professores assumem a responsabilidade de tutoria e se torna responsável por liderar uma equipe de alunos, que passarão a se dedicar em projetos que visam solidificar o conhecimento e contribuir com a comunidade interna e externa. Embora exista um padrão proposto pelo Ministério da Educação (MEC), as universidades podem estruturar seus próprios PETs, como acontece na maioria dos grupos do CEFET-MG, onde a quantidade de alunos de cada grupo, carga horária a ser cumprida e demais detalhes são decididos pela própria instituição.

O PET do curso de Engenharia Elétrica do CEFET-MG Campus Nepomuceno possui um tutor e três cotutores, responsáveis por dez alunos, sendo oito bolsistas e dois voluntários. $\mathrm{O}$ planejamento das atividades é realizado sempre no fim do ano letivo e a execução ocorre ao longo de todo o ano subsequente, respeitando uma carga horária mínima de 20 horas semanais dedicadas por cada aluno petiano. Embora o foco central do grupo seja em atividades relacionados à eficiência energética, outras vertentes também são exploradas.

\section{DESENVOLVIMENTO}

O Programa de Educação Tutorial em Engenharia Elétrica (PETEE) do CEFET-MG Campus Nepomuceno tem por finalidade desenvolver atividades que abrangem a tríade pesquisa, ensino e extensão. Apoiado a essa premissa, o grupo PETEE visa realizar atividades propostas pelos tutores e petianos, que evidenciam o uso consciente e seguro da energia elétrica, além de promover eventos no âmbito social das diversas áreas que circundam o ambiente acadêmico. Este artigo tem o intuito de apresentar algumas das atividades já desenvolvidas pelo grupo.

\subsection{Atividades Desenvolvidas}

\section{Cartilha informativa para os alunos ingressantes no curso de engenharia elétrica}

A entrada em uma universidade é um sonho da maioria dos estudantes que desejam um futuro profissional promissor. Após a euforia de iniciarem essa nova jornada, os estudantes precisam absorver uma grande quantidade de informações sobre o curso, sobre a instituição e, em muitos casos, sobre a cidade. Isso pode se tornar motivo de chateação e estresse para os calouros. Assim, o grupo PETEE Nepomuceno desenvolveu uma cartilha informativa para os alunos que estão ingressando no curso de Engenharia Elétrica. A cartilha contém informações sobre o Campus e também sobre a cidade de Nepomuceno. Desse modo, os alunos que estão iniciando o curso podem esclarecer suas principais dúvidas, ajudando-os assim, a se adaptarem com maior facilidade e conforto nessa nova etapa de suas vidas. 
Figura 1 - Frente e verso da cartilha desenvolvida.

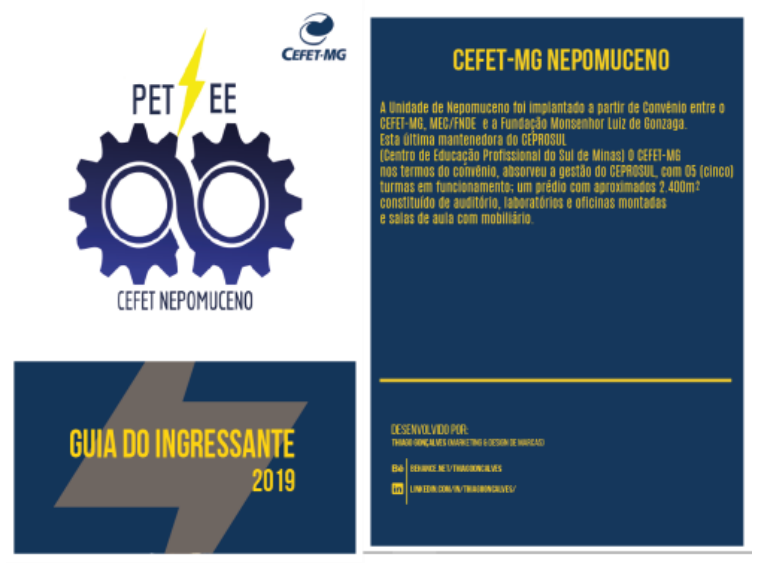

Fonte: Grupo PETEE Nepomuceno.

\section{Projetos de Iniciação Científica}

Ao longo do ano, dois projetos de iniciação científica foram desenvolvidos pelo grupo. Eles tiveram como premissa investigar a tecnologia fotovoltaica e torná-la mais acessível para a comunidade interna e externa do CEFET-MG Campus Nepomuceno. Estes trabalhos, ainda que atrelados, possuem propostas distintas e, portanto, foram realizados de forma independente por duas duplas.

O primeiro projeto, denominado "Dimensionamento de sistemas de geração fotovoltaicos - Estudo direcionado ao município de Nepomuceno-MG", foi desenvolvido pelos petianos Iago Monteiro Vilela e Ludmila Aparecida de Oliveira e orientado pelo co-tutor Ítalo Arthur João Wilson da Silva Meireles. Trata-se de um estudo de caso que se propôs a desvendar a tecnologia fotovoltaica, iniciando com uma descrição do funcionamento da tecnologia e comparação com os demais meios de geração de eletricidade, seguido por uma apresentação do cenário Brasileiro, no que se refere à capacidade de geração disponível e, por fim, realizando um dimensionamento de uma instalação adequada para suprir as demandas de uma residência de médio porte do município. O projeto revelou que é bastante viável a implementação de uma instalação fotovoltaica na região de Nepomuceno-MG, que apresenta uma boa incidência solar ao longo de todo o ano. Entretanto a implementação da tecnologia requer um projeto relativamente complexo, que busca maximizar a geração de energia, diminuir o alto investimento e operar de forma estável e segura.

Já o segundo projeto, intitulado "Estudo comparativo entre os principais componentes de sistemas fotovoltaicos", foi desenvolvido pelas petianas Ana Flávia Rodrigues Lopes e Sara Luiza da Silva e orientado pela co-tutora Juliana Vilela Lourençoni Botega. Esse projeto buscou analisar os diferentes componentes presentes em uma instalação fotovoltaica, focando em suas principais características, diferenças e na disponibilidade de mercado. O trabalho destacou que todos os componentes de uma instalação fotovoltaica estão disponíveis para compra no País. Além disso, pode-se dizer que o mercado fornece todas as informações técnicas consideradas relevantes para uma boa compra, entretanto, em muitos casos estas informações não são 
devidamente observadas, o que pode resultar em um gasto desnecessário ou até mesmo em um projeto problemático.

\section{Desenvolvimento de um sistema de posicionamento de painéis fotovoltaicos}

Este projeto é uma tentativa de otimizar o processo de geração de energia elétrica através de placas fotovoltaicas. A eficiência desse processo é limitada pela intensidade da irradiação solar que está diretamente relacionada com a posição do sol. Quando os módulos fotovoltaicos se encontram parados, recebem diferentes níveis de irradiação solar ao longo do dia. Seu protótipo pode ser observado na Figura 2. Foram criados pelos petianos os diversos sistemas inerentes ao funcionamento do posicionamento automático, tais como: fonte de alimentação do motor, responsável pelo movimento da placa; código em linguagem $\mathrm{C}$, responsável por controlar o motor; produção da placa eletrônica de circuito impresso, entre outros.

Figura 2 - Protótipo do sistema.



Fonte: Grupo PETEE Nepomuceno.

\section{Organização do IV InterPET}

O grupo PET Engenharia Elétrica foi responsável pela organização e realização do IV InterPET - Encontro dos Grupos de Programas de Educação Tutorial do CEFET- MG. O encontro ocorreu nos dias 29, 30 e 31 de agosto de 2019 e contou com a participação de 11 grupos PETs.

O evento tem como principal objetivo a troca de experiências, reflexões, e discussões acerca do desenvolvimento do programa, em especial no que se refere a articulação entre ensino, pesquisa e extensão. Desse modo, o encontro promoveu a disseminação de conhecimento por meio de palestras, oficinas, minicursos e debates. Além disso, o evento proporcionou a troca de experiências e aproximação entre os grupos, através de relatos das atividades que cada grupo desenvolveu.

A organização desse encontro foi de grande importância para todos os membros, contribuindo para um melhor desenvolvimento do grupo. Além do ganho de experiências por organizar um evento de tal importância, o aprimoramento da capacidade dos componentes do PETEE no planejamento e logística na realização de grandes eventos, agregando crescimento pessoal e coletivo para o grupo, também muito relevante. 
Figura 3 - IV InterPET - Encontro dos Grupos de Programas de Educação Tutorial do CEFET- MG.
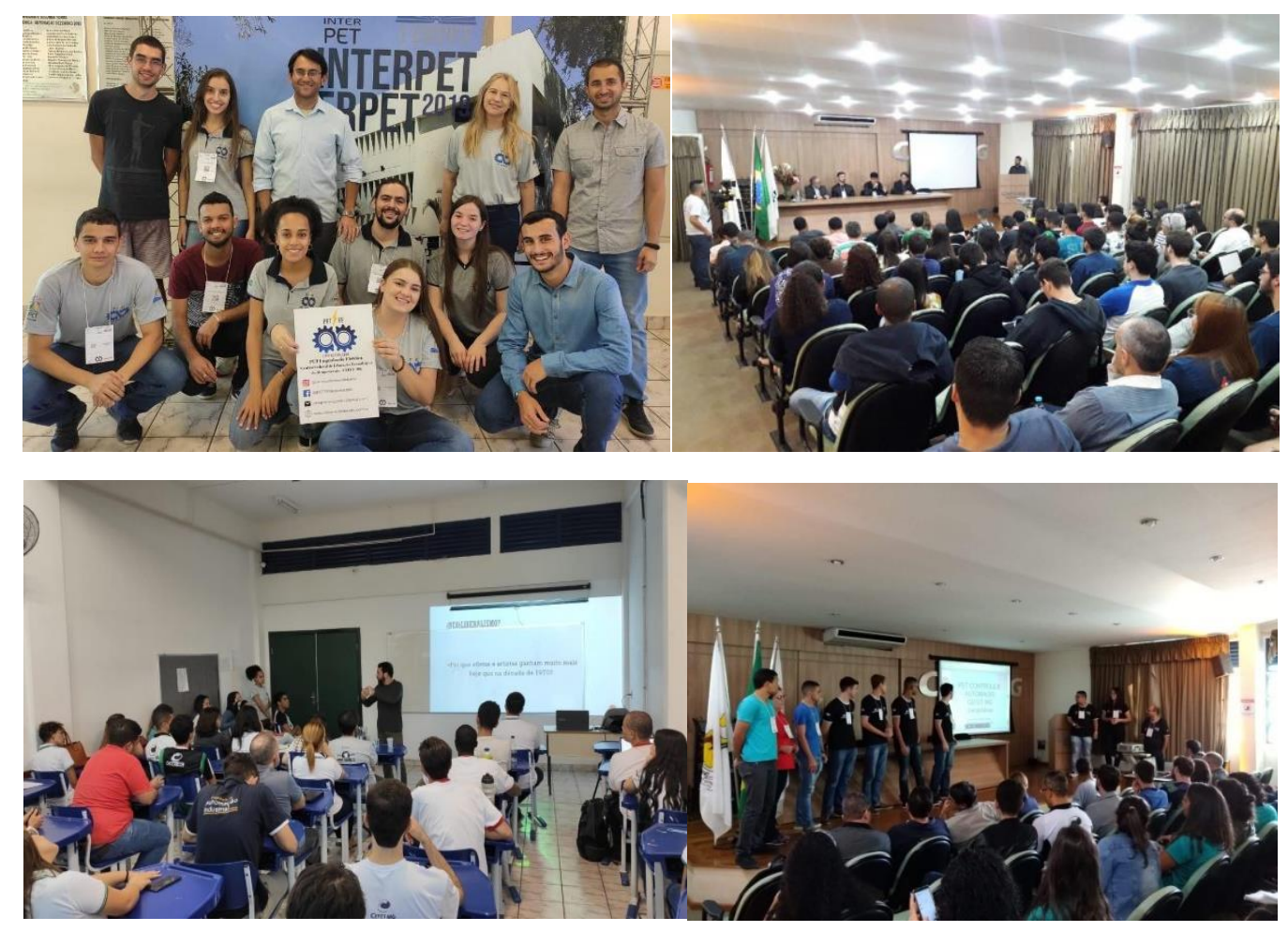

Fonte: Grupo PETEE Nepomuceno.

\section{Organização do I ERPET}

O grupo PET Engenharia Elétrica do CEFET-MG Campus Nepomuceno promoveu, juntamente com o IV InterPET - Encontro dos Grupos de Programas de Educação Tutorial do CEFET- MG, o I ERPET - CEFET-MG, Encontro Regional de Grupos Pets no Campus Nepomuceno. O ERPET é um evento idealizado pelo grupo PETEE, que visa elucidar a importância da tríade ensino, pesquisa e extensão tanto para a comunidade cefetiana quanto para a comunidade externa, contribuindo para reflexão sobre a função social dos grupos como agente transformador fora e dentro da universidade, por meio da elaboração de atividades que promovam a conscientização quanto ao uso eficiente da energia.

Em sua primeira edição, o evento debateu acerca das vertentes do Programa de Educação Tutorial com os grupos PET da região, através da exposição dos trabalhos desenvolvidos por cada grupo. Ademais, o ERPET ofereceu palestras, minicursos e roda de conversa voltada para alunos da rede pública do município, incentivando os estudantes a desenvolverem conhecimento na área, agindo como agentes multiplicadores tanto na escola quanto no ambiente familiar. 
Figura 4 - I ERPET - CEFET-MG - Encontro

Regional de Grupos Pets no Campus Nepomuceno.

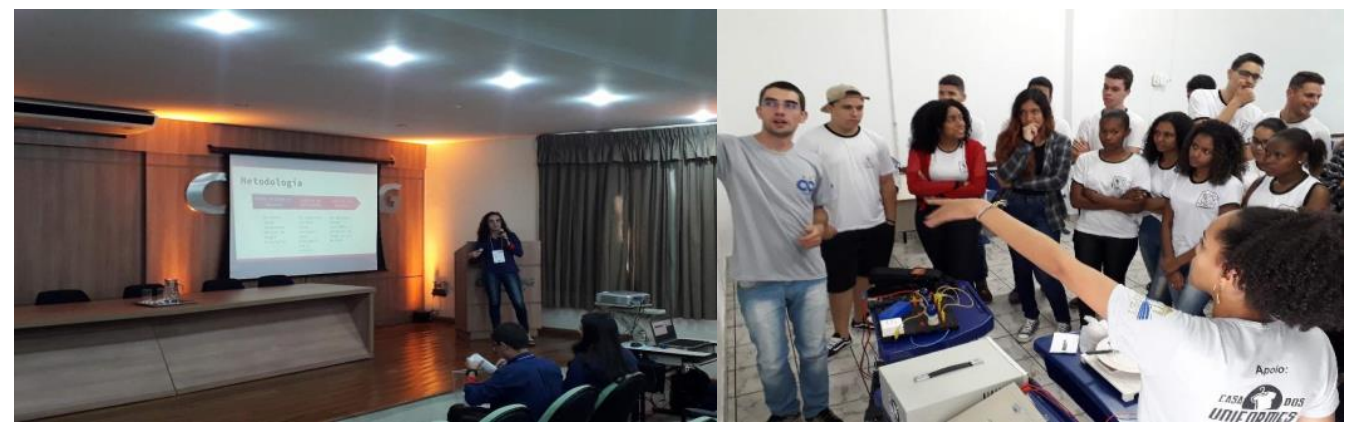

Fonte: Grupo PETEE Nepomuceno.

\section{Participações em eventos}

Percebendo a importância que a troca de experiências apresenta quanto ao desenvolvimento do conhecimento, o grupo PETEE visa participar de eventos regionais e nacionais os quais tenham temas relacionados diretamente ao PET, bem como eventos voltados à área da engenharia elétrica. A seguir são apresentados alguns eventos que contaram com a presença de membros do PET Engenharia Elétrica.

- $15^{\mathrm{a}}$ Semana C\&T-Semana de Ciência e Tecnologia

O grupo PETEE, através de seus membros, ministrou dois minicursos na $15^{\text {a }}$ Semana C\&T. O primeiro minicurso, denominado "Python 3", foi apresentado pelos petianos Breno Masson Lima, Leonardo Silva Ricardino e Ualifer Abreu da Silva. Tal minicurso apresentou uma introdução à linguagem de programação Python, objetivando mostrar as principais características da linguagem e resolvendo exercícios de lógica simples, enfatizando a versatilidade e a importância desta linguagem para o mercado de trabalho futuro. Já o segundo minicurso, chamado "AppInventor com Arduino", foi promovido pelos petianos Ignásia Aline Gama Carvalho Ferreira, Samuel de Souza Ferreira Terra, Letícia Soares Santos e Silfarley Gonçalves. Esse minicurso expôs a aplicação AppInventor, abordando suas formas de utilização, suas funções e como ela pode operar em comunicação com a plataforma Arduino.

Figura 5 - Participação na $15^{\mathrm{a}}$ Semana C\&T.

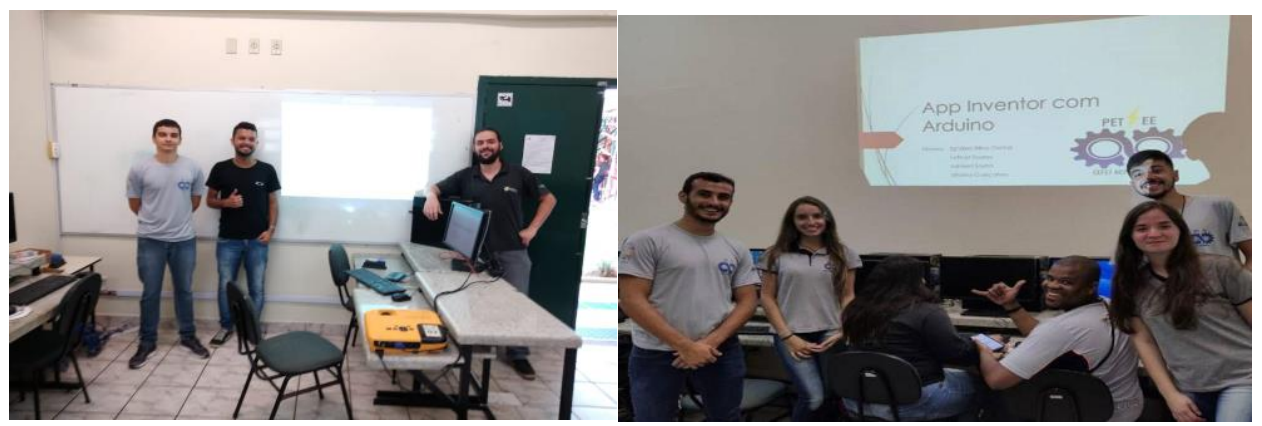

Fonte: Grupo PETEE Nepomuceno. 
- I CONPET- Encontro dos Grupos do Programa de Educação Tutorial da Engenharia Elétrica

O grupo Pet Engenharia Elétrica do CEFET-MG participou do I CONPET, realizado na UFJF (Universidade Federal de Juiz de Fora) em Juiz de Fora - MG. O CONPET é um evento que foi criado para unir a comunidade petiana das áreas afins da Engenharia Elétrica, visando a troca de conhecimento e experiências entre grupos novos e grupos mais antigos, bem como parcerias entre os mesmos. Ao longo do evento, todos os grupos PET apresentaram seus trabalhos nas áreas de ensino, pesquisa e extensão. O evento foi de grande relevância, visto que todos os grupos PET envolvidos puderam ver os projetos desenvolvidos pelo grupo sede e também pelos demais grupos.

Figura 6 - Participação no I CONPET

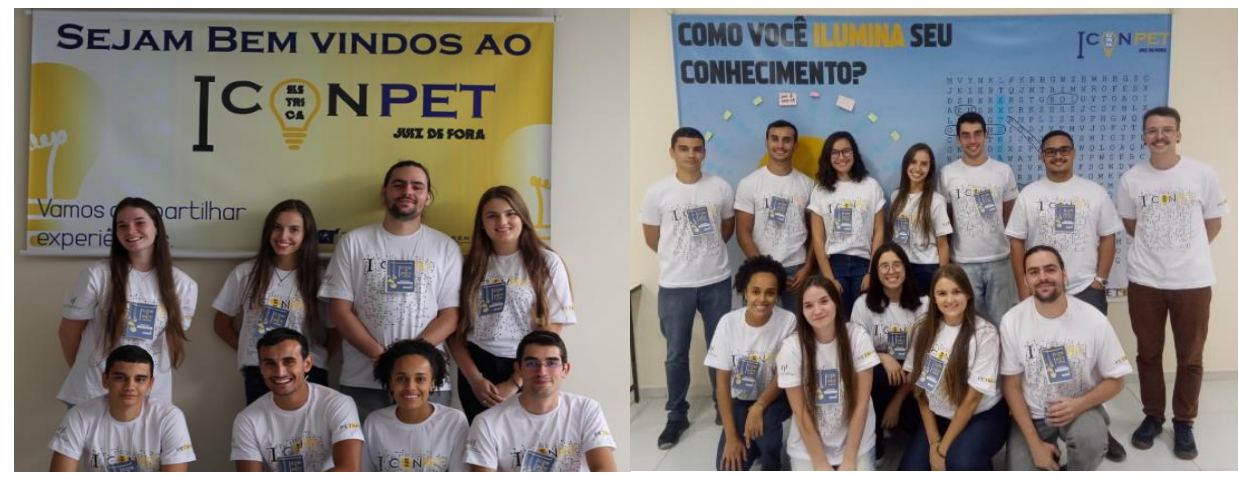

Fonte: Grupo PETEE Nepomuceno.

- Participação nas Feiras de Ciências das escolas públicas de Nepomuceno

O grupo PETEE participou das feiras de ciências das escolas públicas de Nepomuceno. O objetivo é levar ao conhecimento dos alunos, detalhes sobre a instituição, relatando quais são os cursos ofertados pelo CEFET-MG Campus Nepomuceno, além de expor projetos da área de Engenharia Elétrica, visando apresentar o curso para os estudantes. As visitas aconteceram nos sábados letivos das escolas e foram utilizados banners de divulgação e algumas atividades interativas, como o jogo GENIUS.

Figura 6 - Participação nas Feiras de Ciências.

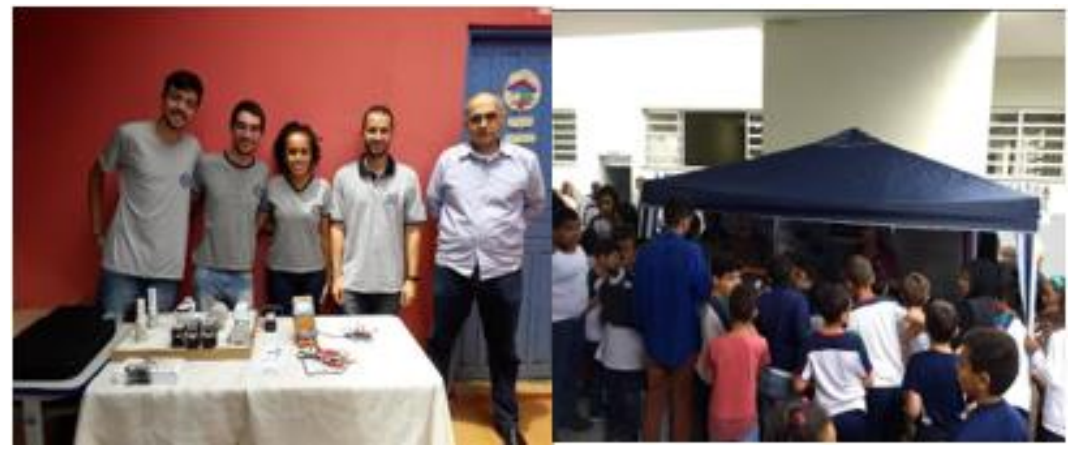

Fonte: Grupo PETEE Nepomuceno. 


\section{Promoção de palestras}

O grupo PETEE busca promover palestras de temas específicos para os estudantes da graduação, bem como para os alunos dos cursos técnicos e para a comunidade externa. $\mathrm{O}$ objetivo é abordar as várias áreas da engenharia elétrica, ampliando assim, o campo de visão dos discentes sobre o curso e aumentando o interesse pela área, além de contribuir para o enriquecimento e complementar os conteúdos vistos em salas de aulas.

Uma das palestras apresentadas pelo grupo, intitulada "Conscientização de Crianças Quanto ao Uso Consciente de Energia”, ministrada pelas petianas Letícia Soares Santos e Ignásia Aline Gama Carvalho Ferreira, promoveu a conscientização quanto ao uso eficiente da energia. Também foi discutido o assunto eficiência energética para o âmbito escolar, incentivando os estudantes a desenvolverem conhecimento na área, agindo como agentes multiplicadores tanto na escola quanto no ambiente familiar.

Figura 7 - Palestra "Conscientização de Crianças Quanto ao Uso Consciente de Energia".

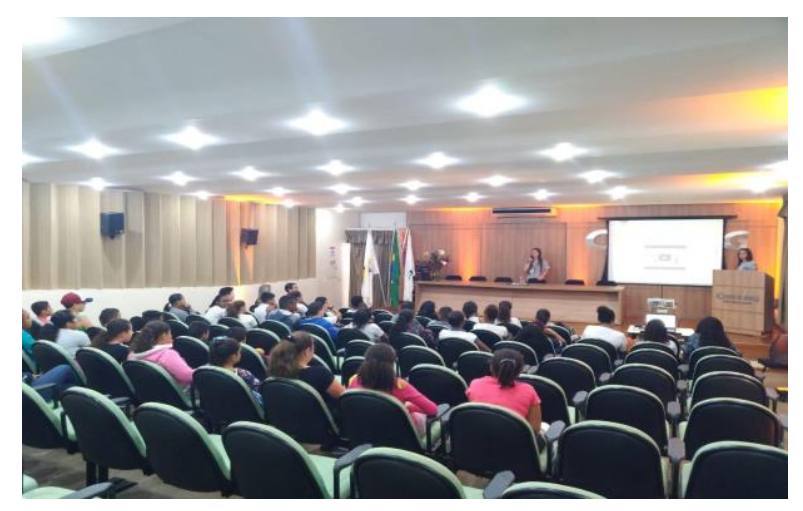

Fonte: Grupo PETEE Nepomuceno.

\section{CONSIDERAÇÕES FINAIS}

As atividades descritas neste trabalho permitem concluir que o grupo PETEE do CEFETMG Campus Nepomuceno, tem conseguido de forma eficiente cumprir seus objetivos e metas no que diz respeito a tríade ensino, extensão e pesquisa.

Os projetos de iniciação científica mencionados estudaram o tema "geração de energia fotovoltaica", que é de alta relevância na área da engenharia elétrica e que está em evidência por se tratar de uma energia limpa, renovável e cada vez mais viável.

Nas atividades relacionadas ao ensino, o grupo conseguiu abranger áreas tecnológicas, como os minicursos de Python e Arduino. Ao mesmo tempo também realizar palestras de conscientização e produzir materiais para instrução de alunos novatos no curso de graduação, algo que pode vir a contribuir na diminuição do índice de evasão nos primeiros períodos.

Tão importante quanto as áreas de ensino e pesquisa, o terceiro eixo, que é a extensão, foi igualmente explorado com projetos de feiras de ciências em outras escolas, levando projetos técnicos, informações sobre os cursos do CEFET-MG, entre outros. Além deste, o I ERPET que contou com palestras de conscientização também possibilitou a extensão das atividades do 
PETEE, utilizando alunos das escolas públicas do município como multiplicadores de conhecimento no ambiente familiar e escolar.

Conclui-se finalmente que o Grupo PETEE tem atuado fundamentalmente, contribuindo com a comunidade interna e externa por meio da produção de diversas atividades relacionadas ao ensino, pesquisa e extensão, sempre pensando no bem comum, no desenvolvimento social e na produção de tecnologias.

\title{
REFERÊNCIAS
}

FILHO, M. F. de F. Resolução CGRAD 35/17, de 05 de julho de 2017 - Revisa o Programa Institucional de Educação Tutorial do CEFET-MG. Disponível em: http://www.dirgrad.cefetmg.br/wp-content/uploads/sites/81/2018/02/Resolucao-CGRAD35_17-Resolu\%C3\%A7\%C3\%A3o-PET.pdf. Acesso em: 12 fev. 2020.

PETEE NEPOMUCENO. O que é o PET Engenharia Elétrica?. Disponível em: https://www.peteenepomuceno.com.br/. Acesso em: 20 fev. 2020.

PORTAL MEC. Apresentação - PET. Disponível em: http://portal.mec.gov.br/pet/pet. Acesso em: 12 fev. 2020.

TOSTA, Rosa Maria et al. Programa de educação tutorial (PET): uma alternativa para a melhoria da graduação. Psicol. Am. Lat., México, n. 8, nov. 2006. Disponível em: http://pepsic.bvsalud.org/scielo.php?script=sci_arttext\&pid=S1870350X2006000400004\&lng=pt\&nrm=iso. Acesso em: 13 fev. 2020.

\section{PET ELECTRICAL ENGINEERING CEFET-MG CAMPUS NEPOMUCENO - BUILDING A SUCCESS MODEL}

\begin{abstract}
Aiming to meet the demands coming from the university and society, the tutorial education program of the electrical engineering course at CEFET-MG Campus Nepomuceno is in its third year of operation. The experience accumulated over that time proved to be fundamental so that in 2019 the group came to present a more ambitious and innovative action plan. Among the activities developed are lectures and short courses, organized events and research projects. Some of these activities already existed before and were rethought so that it could reach a larger audience and better efficiency. The results described here reveal that the performance of the group has been fundamental for the development of the internal and external community of CEFET-MG Nepomuceno.
\end{abstract}

Keywords: PET Group. Electrical engineering. Teaching. Research. Extension. 